This item was submitted to Loughborough's Research Repository by the author.

Items in Figshare are protected by copyright, with all rights reserved, unless otherwise indicated.

\title{
Motivational processes in the coach-athlete relationship: a multi-cultural self- determination approach
}

PLEASE CITE THE PUBLISHED VERSION

https://doi.org/10.1016/j.psychsport.2017.06.004

PUBLISHER

(C) Elsevier

VERSION

AM (Accepted Manuscript)

\section{PUBLISHER STATEMENT}

This work is made available according to the conditions of the Creative Commons Attribution-NonCommercialNoDerivatives 4.0 International (CC BY-NC-ND 4.0) licence. Full details of this licence are available at: https://creativecommons.org/licenses/by-nc-nd/4.0/

\section{LICENCE}

CC BY-NC-ND 4.0

\section{REPOSITORY RECORD}

Jowett, Sophia, James W. Adie, Kimberley J. Bartholomew, Sophie Xin Yang, Henrik Gustafsson, and Alicia Lopez-Jimenez. 2017. "Motivational Processes in the Coach-athlete Relationship: A Multi-cultural Selfdetermination Approach". Loughborough University. https://hdl.handle.net/2134/26581. 
1

2 Objective: Grounded in self-determination theory, the present study examined the cultural

3 invariance of a model that hypothesized sport performers' well-being will be predicted by both their

4 perceptions of motivation and the quality of the relationship held with their coach.

5 Method: Participants ( $\mathrm{N}=756$ ), originating from five countries (British, Chinese, Greek, Spanish,

6 and Swedish), completed a questionnaire that measured perceived coach-athlete relationship quality,

7 basic psychological need satisfaction, self-determined motivation, and well-being. Structural

8 equation modelling (SEM) was employed to analyze the data.

9 Results: Analysis revealed that athletes who perceived a high quality relationship experienced

10 heightened levels of basic need satisfaction. Need satisfaction positively predicted self-determined

11 motivation, which, in turn, linked to enhanced well-being. Moreover, mediation analyses supported

12 the explanatory roles of need satisfaction and self-determined motivation within the model. Lastly,

13 multi-sample SEM invariance testing revealed the model to be largely invariant across cultures.

14 Conclusions: The results support the universal application of self-determination theory and the

15 central role interpersonal relationships play in promoting well-being.

16

17 Key words: Coach-athlete relationships, self-determination, basic psychological needs, motivation, 

Jowett, 2007). Drawing from SDT (Ryan \& Deci, 2002; Vallerand, 2007), and in extending the

11 motivational model of coach-athlete relationships (Magaeu \& Vallerand, 2003), the current study

12 provided a comprehensive test of the motivational processes involved in how perceived

13 coach-athlete relationship quality links to athletes' well-being. Secondly, the cross-cultural

14 invariance of this model was examined across five different countries. self-realization of an individual (athlete) that is optimally functioning, and meaningfully engaged in pursuing their potential in a designated context (sport). To this end, theBasic Psychological Needs 
1 Motivation (HMIEM; Vallerand, 2007) are two prominent SDT frameworks applied to enhance our

2 understanding of coaching behaviors relevant to the motivational processes underpinning the

3 well-being of athletes. .

4

6

7 (DeCharms, 1968); competence, is fulfilled when individuals feel a sense of being effective in the

8 context at hand (White, 1959); and relatedness, is fulfilled when individual's feel a sense of

9 belongingness to and by significant others (Baumeister \& Leary, 1995).

10

11 enhanced well-being, whereas frustration of one or more of these needs is expected to result in

12 ill-being. Basic need satisfaction is not viewed as an automated process; it requires ongoing support

13 from the social influence of significant others (Ryan \& Deci, 2000). In particular, the coach has

14 emerged as a proximal contact in sport settings for influencing the motivation and optimal

15 functioning of athletes (e.g., Jowett \& Shanmugam, 2016; Mallet, 2005). Many BPNT-based sport

16 studies have tested the implications of different perceived coaching behaviors (autonomy support

17 and control) on the motivational processes associated with athletes' well-/ill-being (see Adie \&

Bartholomew, 2013; Taylor, 2015 for reviews).

19

Despite Deci and Ryan’s (2000) assertion that all three psychological needs are essential for 
1 for optimal growth and health to occur; each psychological need has not always independently and

2 consistently predicted indices of well- and ill-being in sport research (e.g., Adie et al., 2012;

3 Quested \& Duda, 2010). Such findings may suggest that one need may have a greater functional

4 significance than another in any given setting (Deci \& Ryan, 1985; Felton \& Jowett, 2013a;

5 Reinboth, Duda \& Ntoumanis, 2004). Alternatively, it may also suggest that other motivational

6 processes, in addition to need satisfaction, may be at play in determining athletes' well-being.

7 The hierarchical model of intrinsic and extrinsic motivation (HMIEM; Vallerand, 2007), a

8 four-stage sequence (social factors $\rightarrow$ basic need satisfaction $\rightarrow$ motivation regulations $\rightarrow$

9 consequences), has also been applied to provide a comprehensive examination of the motivational

10 mechanisms accounting for how coaching behaviors influence athlete well-being (e.g., Álverez,

11 Balaguer, Castillo \& Duda, 2009). According to the HMIEM (Vallerand, 2001), the motivation of an

12 individual (in this case, athlete) is regulated by the degree to which their behavior is more or less

13 self-determined (Ryan \& Deci, 2002, 2007). At the highest end of the self-determination continuum

14 is intrinsic motivation which represents the inherent satisfaction and enjoyment derived from

15 performing an activity (Deci \& Ryan, 1985). Extrinsic motivation is regulated by behavior that is

16 concerned with obtaining aseparate outcome other than participating in the activity itself (Ryan \&

17 Deci, 2000). It is a multi-dimensional construct represented by: integrated (e.g., participating in a

18 behavior that is congruent with one's sense of self), identified (e.g., engaging in an activity because

19 the underlying value of it has been accepted), introjected (e.g., participating in an activity out of 
1 internal pressures), and external (e.g., feeling controlled by external pressures to participate in an

2 activity) forms of regulation (Ryan \& Deci, 2007). Finally, amotivation reflects the complete

3 absence of self-determination in an activity (Deci \& Ryan, 2000).

4 In accordance with the HMIEM, the motivational regulations have been found to have

5 different antecedents and consequences in the extant sport literature (Vallerand, 2007). The majority

6 of research in the sport domain has found support for the supposition that satisfaction of the

7 psychological needs is essential for experiencing more self-determined motivation, whereas

8 frustrating one or more of the psychological needs has resulted in less self-determined motivation or

9 amotivation (e.g., Pope \& Wilson, 2012; Sarrazin et al., 2002). Aligned with theoretical postulates,

10 sport research has also found more self-determined types of motivation (i.e., intrinsic, integrated,

11 identified regulations) to be associated with adaptive consequences (e.g., Álverez et al., 2009;

12 Pelletier, Fortier, Vallerand \& Brière, 2001), whereas for less self-determined types of motivation

13 (i.e., introjected, external regulations), and amotivation, they have been maladaptive (e.g., Calvo et

14 al., 2010; Kowal \& Fortier, 1999).

16 has been evidenced for BPNT (see Adie \& Bartholomew, 2013). Research has emerged to suggest

17 that psychological need satisfaction may not only predict the well-being of competitive athletes

18 directly (Quested et al., 2013), but also indirectly via self-determined motivation (e.g., Álverez et al.

19 2009). Therefore, the HMIEM provides a comprehensive examination of the potential motivational 
1 mechanisms accounting for variance in the well-being of athletes.

2 Collectively, the findings in the sport literature, guided by these two theoretical frameworks,

3 also point towards key coaching behaviors (e.g., autonomy-supportive, task-involving climates) that

4 have consistently been shown to support basic need satisfaction (and self-determined motivation)

5 for the experience of athlete well-being (e.g., Adie \& Bartholomew, 2013; Gagné et al., 2003).

6 Although this line of work holds important implications for theory and practice, it is limited to

7 investigating coach behaviors. A handful of studies (e.g., Felton \& Jowett, 2013a; 2013b) have

8 tested the proposition that the coach-athlete relationship may have the potential to explain

9 motivational processes and well-being in sport settings (see La Guardia \& Patrick, 2008).

10 An Extended Motivational Model of Coach-Athlete Relationships and Well-Being

11 The significance of the coach-athlete relationship for athletes' motivation was emphasized by

12 Mageau and Vallerand (2003). In their motivational model of coach-athlete relationships, three

13 facets of coach behavior (i.e., perceived autonomy support, structure, and involvement) are assumed

14 to predict basic need satisfaction and, in turn, self-determined motivation. The tenets of this model

15 have been empirically supported in the extant literature (Pope \& Wilson, 2012). Nevertheless, these

16 three coaching behaviors are not entirely reflective of the interdependent nature of quality

17 coach-athlete relationships where not only behavioral but also cognitive and affective facets are

18 involved (see Jowett \&Shanmugam 2016). Thus, the application of this model in understanding the 
1 self-determined motivation is limited. It is therefore necessary to consider an extended motivational

2 model of coach-athlete relationships that is conceptualized holistically and can be readily integrated

3 with SDT principles.

4 The coach-athlete relationship is defined as a state in which a coach's and an athlete's thoughts

5 of commitment, feelings of closeness, and complementarity behaviors are mutually interdependent

6 (Jowett, 2007). Commitment refers to the intent of the coach and athlete to form a close and healthy

7 longstanding partnership. Closeness defines the emotional kinship experienced between the dyad

8 members and is characterized by appreciation, trust, and respect for one another. Complementarity

9 reflects the level of co-operation between the coach and athlete and incorporates behaviors such as

10 friendliness, responsiveness, and willingness as well as the key roles each member undertakes in the

11 dyad. The 3Cs model has received empirical support with athletes and coaches of different ages,

12 genders, and levels of performance (e.g., Jowett \& Nezlek, 2012), different types of sport (e.g.,

13 Rhind, Jowett \& Yang, 2012), personality traits (Yang, Jowett, \& Chan, 2015), and different cultural

14 groups (e.g., Yang \& Jowett, 2013).

The 3Cs model of the coach-athlete relationship (see Jowett \& Shanmugam, 2016), is assumed

16 to hold theoretical implications for athletes’ motivation and psychological well-being (Jowett, 2007).

17 To date, seldom sport research exists linking coach-athlete relationship variables to motivational processes and subsequent well-being. This is surprising given Jowett $(2005,2007)$ has posited that 
1 be expressed and fulfilled. Although still in its infancy, SDT research has emerged to find that

2 perceived coach-athlete relationship quality (as operationalized by the 3 C's), serves as important

3 determinants of basic need satisfaction (Choi et al., 2013, Felton \& Jowett, 2013b; Riley \& Smith,

4 2011), self-determined motivation (Riley \& Smith, 2011), and well-being (Felton \& Jowett, 2013b). Riley and Smith (2011) are credited as the first researchers to test Mageau and Vallerand’s

6 (2003) model incorporating a relationship perspective. They found the three psychological needs to

7 mediate the positive association between perceived coach-athlete relationship quality (as defined by

8 the 3 C's) and self-determined motivation among young sport participants. In testing BPNT, Felton

9 and Jowett (2013a, 2013b, 2015) conducted a series of studies demonstrating the predictive utility

10 of social relationships in sport (i.e., secure coach-attachment, perceived coach-athlete relationship

11 quality) for satisfaction of the basic psychological needs and subsequent well-being among adult

12 athletes. One novel contribution of this work, in following the approach of relationship researchers

13 in SDT (e.g., La Guardia et al., 2000; Patrick et al., 2007), is that the findings are based on need

14 satisfaction within one's relationship. On the basis that coaches' contribution is instrumental in

15 organized sport, and the quality of the relationship they develop with their athletes forms a platform

16 for experiencing basic need satisfaction, we too deemed it necessary to study relationship need

17 satisfaction. Guided by and extending past research (e.g., Jowett \& Felton, 2013b; Riley \& Smith,

18 2011), our study aimed to provide a more comprehensive model of the motivational processes 
1 Vallerand's work specifically (e.g., Mageau \& Vallerand, 2003; Vallerand, 2007), this study is the

2 first to test the full sequence of associations between perceived coach-athlete relationships,

3 relationship need satisfaction, motivation and well-being in sport among competitive athletes.

4 The Universality Concept of SDT

According to SDT (Ryan \& Deci, 2002), the basic psychological needs are postulated to be

6 universal regardless of age, gender, setting or culture. In the sport domain, there have been very

7 limited cross-cultural studies testing this universality concept. Quested et al. (2013) conducted and

8 found support for invariance of a model predicting basic need satisfaction, enjoyment and intention

9 to drop out of sport among youth soccer players across 5 European countries. Taylor and Lonsdale

10 (2010) also found invariance of a BPNT model predicting vitality and effort across Chinese and UK

11 physical education students. To date, no studies have tested the assumed cross-cultural invariance in

12 the hypothesized relationships between perceived coach-athlete relationships, relationship need

13 satisfaction, motivation, and well-being among competitive athletes. Inaddressing this gap in the

14 literatureour cross-cultural study is the first to test the motivational processes associated with the

15 links between perceived coach-athlete relationship quality and well-being among athletes across

16 five countries. It may generate understanding concerning how the relationship with one’s coach, and

17 not merely the influence of their coaches' behaviour, could potentially impact the motivational

18 processes linked to well-being regardless of the cultural background of the athlete. This could

19 suggest the universal importance of developing quality coach-athlete relationships in order to foster 
1 meaningful participation and optimal functioning in competitive sport.

2 The Present Study: Aims and Hypotheses

In proposing an extended motivational model of coach-athlete relationships (Jowett, 2007,

4 Magaeu \& Vallerand, 2003) in the form of the HMIEM (Vallerand, 2007), the first aim of the study

5 was to test a hypothesized model with a four stage sequence: coach-athlete relationship quality $\rightarrow$

6 relationship psychological needs $\rightarrow$ self-determined sport motivation $\rightarrow$ well-being among

7 multi-national sport participants. Based on the tenets of the HMIEM (Vallerand, 2007), and guided

8 by past research (e.g., Riley \& Smith, 2011; Felton \& Jowett, 2013a, 2013b), perceived

9 coach-athlete relationship quality was theorized to positively predict athletes' basic need satisfaction

10 within their relationship. Relationship need satisfaction was expected to positively correspond to

11 self-determined motivation, which in turn, would lead to enhanced well-being.

The second aim of the study was to test the mediational role of relationship need satisfaction

13 and self-determined motivation in the hypothesized model across the five countries. Drawing from

14 the HMIEM (Vallerand, 2007), and guided by past sport research (e.g., Álverez et al., 2009; Riley

15 \& Smith, 2011), it was proposed that relationship need satisfaction would directly mediate the link

16 between perceived coach-athlete relationship quality and self-determined motivation. Based on

17 BPNT (Ryan \& Deci, 2002) and also the HMIEM (Vallerand, 2007), the present study also aimed to 
1 the optimal functioning of athletes.

2 A final aim of the study was to test the cultural invariance of the hypothesized model. Ryan

3 and Deci (2002) assert that whilst the needs themselves are hypothesized to be universal, and the

4 relations between the psychological needs, self-determined motivation, and well-being should apply

5 across cultures (Deci, et al., 2001; Taylor \& Lonsdale, 2010), the means through which the

6 psychological needs are satisfied may vary as a function of culture (Ryan \& Deci, 2000). Based on the

7 assumption that the coach-athlete relationship, as evidenced by the 3 C's, is also assumed to be a

8 universal phenomenon (Jowett \& Yang, 2012), we also expected high quality coach-athlete

9 relationships to positively predict relationship need satisfaction, although the amount of variance

10 explained in this outcome may differ across cultures. Furthermore, despite potential differences in

11 mean levels, perceived support for relationship psychological needs fulfilled by high quality

12 coach-athlete relationships should be positively associated with both optimal motivation and

13 well-being in all cultures, particularly given that needs do not have to be valued explicitly within a

14 particular culture to have functional import (Doyal \& Gough, 1991). In sum, we expected our

15 hypothesized mediational model to remain invariant for competitive athletes from different cultural

16 backgrounds.

Methods

\section{Participants and Procedures}


$1 \quad 50.30 \%$ male), Greece $(\mathrm{N}=168$; Mean age = $19.84(S D=5.78) ; 76.8 \%$ male $)$, Spain $(\mathrm{N}=137$;

2 Mean age $=23.46(S D=4.67) ; 80.9 \%$ male $)$, Sweden $(\mathrm{N}=171$; Mean age $=16.78(S D=1.04)$;

$3 \quad 61.4 \%$ male), and UK ( $\mathrm{N}=137$; Mean age $=22.45(S D=4.53) ; 62.00 \%$ male), voluntarily

4 participated in this study. Athletes competed in 40 different sporting events (length of participation

$5=7.84$ years; $S D=4.63)$, and received training $($ mean training hours $=11.79$ per week; $S D=6.29)$

6 regularly from their coaches (mean length of relationship $=2.07$ years; $S D=2.52$ ). Before data

7 collection, approval was obtained from the lead authors' University Ethics Committee. After

8 obtaining informed consent, participants were asked to complete a multi-section questionnaire

9 measuring relationship quality, relationship need satisfaction, motivation, and well-being.

10

11

12

13

14

15

16

17

18

19

\section{Measures}

Coach-Athlete Relationship Questionnaire (CART-Q). The CART-Q (Jowett \& Ntoumanis, 2004) assesses athletes’ perceptions of the quality of the relationship they have with their coaches within three aspects: closeness ("I trust my coach”; 4 items); commitment ("I am committed to my coach”; 3 items); and complementarity (“I am responsive to his/her efforts”; 4 items). These 11 items were responded to on a 7-point Likert-scale with anchors ranging from "strongly agree” (7) to “strongly disagree” (1). We adopted the translated versions of the scale for Chinese, Greek, Spanish and Swedish participants. Psychometric tests from previous studies supported the validity and reliability of the CART-Q across these countries (e.g., Yang \& Jowett, 2012; Balduck, Jowett, \& Buelens, 2011), and the internal reliability of the closeness ( $\alpha=.86-.93)$, commitment $(\alpha=.74$ 
1 competent person”), 3 autonomy items ("I feel free to be who I am”), and 3 relatedness items (“I

- .89), and complementarity ( $\alpha=.79$ - .88) subscales was good in the present study. Given the strong inter-correlations between the 3C's (Jowett \& Ntoumanis, 2004), and aligned with past SDT research (Riley \& Smith, 2011; Jowett \& Felton, 2013a,b), we simplified our model by using an overall measure of coach-athlete relationship quality, as indicated by the three CART-Q dimensions.

Basic Need Satisfaction in Relationship Questionnaire (BNRSQ). The BNSRQ (La Guardia

et al., 2000) measures the satisfaction of the 3 basic psychological needs within a particular

relationship (in this case, the coach-athlete relationship). The stem used in the questionnaire was

“when I am with my coach" and athletes responded to 9 items, 3 competence items ("I feel like a

10 feel loved and cared about”). Respondents reported how true the items were on a 7-point

11 Likert-scale ranging from "Very true" (7) to "Not true at all” (1). The scale has previously

12 demonstrated sound discriminant and predictive validity as well as good reliability. Following

13 Hambleton's (2005) recommendations, the original scale was translated into Chinese, Greek,

14 Spanish, and Swedish for athletes of these countries to complete. The autonomy $(\alpha=.74-.88)$,

15 competence $(\alpha=.71-.81)$, and relatedness $(\alpha=.62-.83)$ subscales demonstrated acceptable levels

16 of internal reliability. On the basis that the three needs are inter-related constructs (Deci \& Ryan,

17 2000) and in line with past research (e.g., Quested et al., 2013), we formed a single composite variable of relationship need satisfaction using the three subscales as indicators. 
1 Rose, 2008) assesses each of the behavioral regulations on SDT's self-determination continuum by

2 asking why participants (athletes) participate in their sport. Each item started with a common stem

3 "I participate in my sport..." and participants rated how true the items were for them on a 7-point

4 Likert-scale ranging from "Very true" (7) to "Not true at all" (1). The 6 dimensions of the BRSQ

5 include intrinsic motivation (“because it’s fun”; $\alpha=.77-.94$ ), integrated regulation ("because what

6 I do in my sport is an expression of who I am”; $\alpha=.71-.86$ ), identified regulation ("because the

7 benefits of sport are important to me”; $\alpha=.67-.84$ ), introjection (“because I would feel guilty if I

8 quit”; $\alpha=.79-.85$ ), external regulation (“because people push me to play”; $\alpha=.78-.86$ ), and

9 amotivation ("but I question why I continue”; $\alpha=.81-.91$ ) and are assessed by 24 items (4 items for

10 each dimension). The BRSQ was originally developed in English and has been shown to have

11 robust psychometric properties (Lonsdale et al., 2008). A Chinese translated version of the scale has

12 also demonstrated good reliability in previous studies (Chan, Hagger, \& Spray, 2011). Thus the

13 English and Chinese versions of the scale were adopted and the scale was translated into Greek,

14 Spanish and Swedish following the aforementioned procedure. To reduce the complexity of the

15 model, we computed a relative autonomy index (RAI) from the scores of the 5 BRSQ dimensions

16 which represented athletes' overall levels of self-determined motivation. This approach has been

17 widely used in SDT-based sport research (Álverez et al., 2009). Moreover, Sheldon and colleagues

18 (2015) confirmed the validity of the RAI as a suitable assessment of self-determination levels.

19 Therefore, following the guidelines outlined by Lonsdale et al. (2009), we assigned different 
1 weightings to each type of motivation: intrinsic motivation (+2), integrated motivation $(+1)$,

2 identified motivation (+1), introjection (-1), and external motivation (-2). The RAI was

3 subsequently computed by summing the weighted-item-scores. Four RAI indicators were derived

4 from the 20 BRSQ items.

6 al., 2007) was developed to measure well-being, a key indicator of mental health. The 14-item

7 measure assesses the following well-being dimensions: affective-emotional ("I've been feeling

8 good about myself”), cognitive-evaluative (“I’ve been dealing with problems well”) and

9 psychological functioning (“I’ve had energy to spare”). Participants rated how often they

10 experienced the feeling or emotion described in the statement on a 5 point Likert-scale ranging from

11 “All the time” (5) to "None of the time” (1). The original scale was translated into Chinese, Greek,

12 Spanish, and Swedish so that athletes of these countries could complete the multi-section

13 questionnaire. Consistent with the evidence of the convergent validity provided during the scales

14 validation, the reliability indices of the WEMWBS were high $(\alpha=.84-.92)$ in the present study. To

15 reduce the complexity of the model, the items were parceled to form 5 well-being indicators.

16 Analysis 
1 root-mean square error of approximation (RMSEA) and standardized root-mean square residual

2 (SRMR) were lower than .08 (Hu \& Bentler, 1999). Comparative fit index (CFI) and Tucker Lewis

3 index (TLI) greater than .95 was indicative of very good fit (Hu \& Bentler), with values greater

4 than .90 interpreted as adequate fit (Bentler, 1990).

6 between the latent factors) was tested to examine the factor structure and reliability of measurement

7 across the data drawn from the 5 countries. After ensuring the measurement models demonstrated a

8 good fit to the data, single-group structural models were employed (various factor-to-factor paths

9 were inputted for hypotheses testing). Finally, multi-group SEMs were carried out to examine the

10 invariance of the measurement model and structural model across the 5 countries.

11 Single-group SEM. For the data set from each country, we freely estimated the factor

12 paths in the hypothesized model (i.e., coach-athlete relationship quality $\rightarrow$ psychological need

13 satisfaction $\rightarrow$ self-determined motivation $\rightarrow$ well-being). We also investigated whether mediation

14 existed in the hypothesized associations. Specifically, the following indirect effects were examined:

15 (a) coach-athlete relationship quality on self-determined motivation via need satisfaction (b) need

16 satisfaction on well-being via self-determined motivation and (c) coach-athlete relationship quality

17 on well-being via both need satisfaction and self-determined motivation. Mediation was evidenced

18 when (a) the independent variable (coach-athlete relationship) and the mediator (motivational

19 processes) had significant direct effects on the dependent variable (well-being), (b) the direct effect 
1 of the independent variable on the dependent variable was not significant after controlling the effect

2 of the mediator (Baron \& Kenny, 1986), and (c) the indirect effect of the independent variable on

3 the dependent variable was significant (Zhao, Lynch \& Chen, 2010).

$4 \quad$ Multi-group SEM. First, a baseline multi-group model with factor paths and loadings freely

5 estimated across the 5 countries was tested. Once the model demonstrated an acceptable fit to the

6 data, we further tested the invariance of the hypothesized model by systematically constraining the

7 factor loadings and then the factor paths to be equal across the 5 countries (Byrne, 2006). When the

8 constraints decreased the model fit by a CFI value of more than .010 , it was concluded that the

9 hypothesized model was not invariant across culture. In addition, the Lagrange Multiplier test (LM

10 test) was employed to examine if certain constraints were responsible for the decrease of model fit.

11 If the decrease of the model fit became less than .010 after releasing the paths indicated by the LM

12 test, we concluded that the hypothesized model was partially invariant.

Results

\section{Preliminary Analysis}

The data sets from the 5 countries were screened and there appeared to be no significant

16 multivariate and univariate outliers $(p>.05)$ nor an apparent pattern of missing data $(<1 \%)$.

17 Therefore, the missing values were replaced using the expectation maximization algorithm. CFA of

18 the 5 data sets yielded satisfactory goodness of fit (CFI $>.92$, TLI $>.90$, RMSEA $\leq .08$, SRMR

$19=.08$; full model fit indices are presented in Table 1), which supported the factor structure and 
1 convergent validity of the measures used in the study. The correlation matrix and descriptive

2 statistics are displayed in Table 2.

3 Single-group SEM

4 Apart from the Chinese sample $(\mathrm{CFI}=.904, \mathrm{TLI}=.882, \mathrm{RMSEA}=.101$, SRMR $=.093)$ which

5 demonstrated a mediocre fit, the hypothesized model fitted the data acceptably well for all other

6 countries $(\mathrm{CFI}>.921$, TLI $>.903$, RMSEA $<.071$, SRMR $<.088$; full model fit indices are

7 presented in Table 1). The structural path estimates were in line with our hypothesis across the 5

8 data sets: positive associations were observed in the relationships between (a) coach-athlete

9 relationship quality and need satisfaction, (b) need satisfaction and self-determined motivation, and

10 (c) autonomous motivation and well-being (all $p<.05$; details are displayed in Table 3). Mediation

11 analyses also supported the explanatory role of need satisfaction and self-determined motivation

12 within the hypothesized model, however partial mediations presented in some of the data sets which

13 implied that the independent variables involved in these mediations were likely to have both direct

14 and indirect effects on the dependent variables (see Table 4).

\section{Multi-group SEM}

The baseline multi-group model as well as the models with constrained factor paths and

17 loadings all demonstrated a satisfactory fit to the data. Constraining the factor loadings resulted in a change in the CFI value of .009 and further constraining the structural paths led to a reduction in the

19 CFI of .006 (see Table 5). Therefore, we concluded that the measurement and structural model were 
1 invariant across culture.

2

3

4

6

7 satisfaction within their relationship. Furthermore, relationship need satisfaction predicted higher

8 levels of self-determined motivation, which in turn, led to enhanced well-being. Mediation analyses

9 provided partial support for the explanatory roles of need satisfaction and self-determined

10 motivation within the hypothesized model. Finally, given the multi-national nature of competitive

11 sport, the integrated model was shown to be invariant across the five nations examined. These

12 results provide support for the universal application of self-determination theory and corroborate the

13 integration of a coach-athlete relationship and motivational model.

\section{Beyond Perceptions of Coach Behavior} Jowett, 2013a,b, 2015; Riley \& Smith, 2011), perceived coach-athlete relationship quality positively

17 predicted athletes' basic need satisfaction within their relationship. Whilst limited previous research has examined the impact of closeness, commitment, and complementarity on motivation (e.g., Riley 
1 coach-athlete relationship quality as defined by the 3C's, to SDT-based motivational regulations and

2 provides support for the notion that interpersonal relationships have motivational significance in

3 this context cross-culturally. Using the 3C's model to examine athletes' perceptions of the mutual

4 and causal interconnections between themselves and their coach's feelings, thoughts, and behaviors

5 (Jowett, 2007), this study extends previous SDT-based research beyond simply examining the

6 impact of coach behaviors (see Adie \& Bartholomew, 2013). The focus on what one person does to

7 another may not accurately reflect what goes on between coaches and their athletes (Jowett, 2005,

8 2007) and the generated findings provide evidence that the quality of the coach-athlete relationship

9 characterized by care, commitment, and collaboration are strong predictors of motivation and

10 well-being via the satisfaction of psychological needs. Furthermore, it is recognized that successful

11 coach-athlete relationships can take many different forms that of adaptability, flexibility and

12 accessibility as what one athlete wants and needs from a coach can be very different to what another

13 athlete wants or needs (Jowett \& Shanmugam, 2016). Ultimately, coach-athlete relationships must

14 be meaningful at the personal and cultural level if they are to promote optimal motivational

15 processes and well-being (Jowett \& Yang, 2012).

17 Shanmugam, 2016) proposition that the coach-athlete relationship is a medium through which the needs of dyad members' can be expressed and, in turn, fulfilled. On average, athletes’ perceptions of overall relationship quality were high and equivalent across all five nations. However, whilst 
1 athletes' perceptions of the coach-athlete relationship explained a large amount of the variation in

2 perceptions of need satisfaction among Greek, Swedish and Spanish sub-samples, a smaller amount

3 of variance was explained in Chinese and British sub-samples. Future research should attempt to

4 explain these differing findings by examining the separate components of closeness, commitment,

5 and complementarity in the coach-athlete relationship in order to examine their independent effects

6 on need satisfaction across culture. For example, it may be that perceptions of commitment and

7 closeness play a key role in supporting the psychological needs of Greek athletes and

8 complementarity plays a key role in explaining the variance in need satisfaction scores among

9 Swedish athletes (cf. Jowett \& Yang, 2012). In addition, examining the impact of coach-athlete

10 relationship quality on each of the three needs separately may offer further insight into this

11 relationship. For example, Jowett and Felton (2013b) found that British athletes' perceptions of

12 quality coach-athlete relationship predicted levels of competence but not autonomy or relatedness. Is

13 this link a reflection of the purposeful task-focused nature of the coaching relationship (Jowett \&

14 Shanmugam, 2016) or a cultural effect: Would this finding hold across countries or would it be

15 specific to some? Would closeness be a stronger predictor of relatedness need satisfaction while

16 complementarity a stronger predictor of competence across cultures or within one particular culture

17 (Greece and Sweden respectively). Thus, future research looking at the individual dimensions of the

183 C's and needs satisfaction in relation to athlete motivation is warranted. 
In line with SDT, relationship need satisfaction was expected to positively correspond to

2 self-determined motivation, which in turn, was hypothesized to lead to enhanced well-being. In line

3 with previous research (e.g., Gagné et al., 2003; Kowal \& Fortier, 1999), the present findings

4 supported this model in each national sub-sample however variations among the size of the

5 pathways and the amount of variance explained in the outcome variables were observed. For

6 example, basic psychological need satisfaction explained a large amount of the variance in

7 self-determined motivation among Chinese athletes, a moderate amount among Greek and Swedish

8 athletes, and a small amount among Spanish and British athletes. As mentioned earlier future research

9 should distinguish between the three psychological needs in order to examine whether one has greater

10 functional significance for motivation and well-being in a given culture (e.g., relatedness in

11 collectivistic cultures). Furthermore, whist higher levels of self-determined motivation predicted

12 well-being across the board, relative autonomy only explained a moderate (among Chinese, Greek,

13 Swedish athletes) to low (among Spanish and British athletes) amount of the variance in this

14 outcome. SDT views concerning the universal significance of autonomously regulated behaviors have

16 been much debated. For example, Oishi (2000) argued that greater autonomy only yields benefits to

17 people inside a few highly individualistic Western nations where autonomy is valued (e.g., Sweden, Britain). Similarly, Miller (1999) argued that lack of autonomy is not likely to be detrimental within

19 cultures based in authority or tradition (e.g., China, Greece). In contrast, the current findings 
1 indicated that self-determined motivation had a greater influence on well-being among Chinese and

2 Greek athletes and a smaller impact on the well-being of Spanish and British athletes. Whilst this

3 apparent reversal in terms of what would ordinarily be expected among collectivistic and

4 individualistic cultures is interesting (see Hofstede, 2001), it is important to note that the present

5 findings support previous research (e.g., Sheldon et al, 2004; Taylor \& Lonsdale, 2010) and indicate

6 that self-determined motivation is associated with well-being in all of the countries and cultures

7 examined.

Thus, our findings provide strong support for the universal nature of the psychological needs

9 and the motivational processes outlined in SDT. However, researchers and theorists continue to

10 debate the cross-cultural importance of autonomy and these arguments often center on the way in

11 which 'autonomy' is interpreted (Kagitcibasi, 1996). It is likely that the arguments proposed by

12 Oishi (2000) and Miller (1999) are based upon an interpretation of autonomy as individualism or

13 independence (Chirkov, Ryan \& Willness, 2005). It is, therefore, important to note that SDT

14 explicitly differentiates autonomy (being volitional or endorsing one’s goals and actions) from

15 individualism (cultures which focus on the needs of the individual rather than the needs of the

16 collective) and independence (being separate from others). Thus, one can be autonomously

17 interdependent. This is important given that the coach-athlete relationship is defined as a state in

18 which coaches' and athletes' feelings, thoughts and behaviors via the 3Cs are mutually

19 interdependent (Jowett, 2007). Interdependence Theory (Kelley \& Thibaut, 1978) explains that 
1 good quality interpersonal relationships are associated with rewards (satisfaction and gratification)

2 and poor quality relationships with costs (dissatisfaction and punishment), while SDT views

3 interdependence (mutual/shared dependence) as lying between two extremes of dependence

4 (complete reliance on the other) and independence (complete separation from other). The notion of

5 interdependence provides an opportunity to nurture one's autonomy in the knowledge that there is

6 always a trustworthy partner nearby to support when need be. It would appear that athletes who find

7 themselves in interdependent or good quality coaching relationships, act with a sense of volition,

8 experience an elevated well-being irrespective of cultural and personal preferences.

9 Given the postulates of SDT (Ryan \& Deci, 2002) and the HMIEM (Vallerand, 2007), another

10 reason for the relatively low explanatory power of self-determined motivation could be because the

11 psychological needs also have a direct effect on well-being and thus explain some of the variation in

12 this outcome (Adie et al., 2012). Support for this argument comes from the results of the mediation

13 analysis in which the direct effect of relationship need satisfaction was consistently stronger than the

14 indirect effect of the needs via self-determined motivation. Furthermore, mediational evidence

15 observed in the current study indicated that need satisfaction had a direct and indirect effect on

16 well-being in Greek and Spanish samples. In line with previous research (e.g., Álverez et al., 2009;

17 Balaguer et al., 2012), this finding provided support for the proposition that self-determined 
Need satisfaction was also expected to mediate the link between coach-athlete relationship

2 quality and self-determined motivation. However, whilst this hypothesis was supported among

3 Chinese, Spanish, and Swedish athletes, need satisfaction only partially mediated the link between

4 relationship quality and self-determined motivation in British and Greek sub-samples. These partial

5 mediations indicated that the coach-athlete relationship had a direct influence on well-being as well

6 as impacting on it indirectly via self-determination. Furthermore, partial mediations also indicated

7 that perceived coach-athlete relationship quality had direct and indirect effects on athlete well-being

8 (Chinese, Greek, and Spanish). These findings highlight the influential role of the coaching

9 relationship in performance environments (Jowett 2005; Jowett \& Shanmugam, 2016).

11 mediational model. Based on the universality hypotheses (Ryan \& Deci, 2002) and past research

12 (Jowett \& Yang, 2012; Taylor \& Lonsdale, 2010), it was hypothesized that the proposed model

13 would remain equivalent across cultures. Invariance testing supported this expectation and revealed

14 that the factor loadings (the measurement model) and structural paths (the structural model)

15 remained equivalent across culture. Thus, regardless of culture, the more athletes' perceive the

16 coach-athlete relationship to support their basic psychological needs, the more self-determined their

17 motivation, and the greater their well-being. These findings provide substantial support for SDT's

18 prediction that the impact of need support is universally positive for optimal functioning and

19 well-being because it fits with the intrinsic growth tendencies inherent in human nature (Grolnick, 
1 2003; Ryan \& Deci, 2002). Thus, the current research has supported the integration of a

2 coach-athlete relationship and motivational model which has universal appeal and can, therefore,

3 generate research findings that should be generalizable across cultures and countries.

\section{$4 \quad$ Practical Applications}

The current research has the potential to supply key stakeholders including coaches and sport

6 psychology consultants with sound theoretical knowledge and empirical evidence to assess the

7 quality of a key relationship in an increasingly multi-cultural coaching context (Gill, 2007).

8 Furthermore, the current findings suggest that the coach-athlete relationship has important links to

9 athlete motivation and well-being which are equivalent across cultures. A major advantage of the

10 3C's model is its emphasis on the bidirectional nature of the relationship which is manifested

11 through the construct of co-orientation (Jowett, 2007; Jowett \& Lorimer, 2013). This construct

12 contains two sets of interpersonal perceptions: direct perceptions (I trust my coach/athlete) and

13 meta-perceptions (My coach/athlete trusts me). The measurement of the 3C's and co-orientation can,

14 therefore, be used to analyze coach-athlete dyads and identify areas of dissimilarity or disagreement

15 across the closeness, commitment, and complementarity dimensions. Thus, future research and

16 applied practice should consider obtaining both athletes and coaches direct and meta-perceptions of

17 the coach-athlete relationship. This will enable problem areas to be identified and should result in

18 improvements in the way that coaches and athletes relate, communicate, and interact with each 
1 motivation and well-being among athletes of all nationalities.

\section{Limitations and Additional Future Directions}

Whilst the current research provides new insight into how perceptions of coach-athlete

4 relationship quality affect SDT-based motivational processes and athlete well-being, it is important

5 to acknowledge some limitations. First, the cross-sectional nature of the study limits our ability to

6 infer causal relations between the variables. It is possible, therefore, that athletes' well-being

7 influences how they perceive their relationship with their coach. Thus, whilst there is theoretical

8 and empirical support in the SDT literature for the present study’s proposed sequence (e.g., La

9 Guardia \& Patrick, 2008; Jowett \& Felton, 2013b), research adopting a longitudinal design with the

10 opportunity to investigate changes in the coach-athlete relationship over time and both within- and

11 between-subject relations is an important direction for future research. Second, whilst the exclusive

12 use of self-report measures was primarily predetermined by the nature of the variables included in

13 the theoretical model, the use of more objective indicators of well-being would definitely benefit

14 future research (Bartholomew et al., 2011b). Third, our study was limited to predicting the

15 well-being of athletes. It is also important that future research examines how coach-athlete

16 relationship quality is related to ill-being among athletes (e.g., overtraining and burnout). Adopting

17 direct measures of psychological need thwarting (Costa, Ntoumanis, \& Bartholomew, 2015) may be

18 particularly helpful in this respect.

19 Fourth, we used the RAI (see Ryan \& Connell, 1989) to form a single variable representing 
1 self-determined behavior in the present study. Despite supporting our hypothesized model across

2 culture, it is possible that using the RAI may have masked over the unique predictive effects of the

3 different motivational regulations that make-up the full self-determination continuum. In light of

4 this debate (see Chemolli \& Gagné, 2014), future studies may consider replicating our model using

5 each of the motivational regulations. Fifth, and drawing parallels to the work by Quested et al.

6 (2013), there was over-representation of males in our sample except for one country (China). To

7 help verify past and present findings, we propose that future studies testing the cultural invariance

8 postulate in SDT recruit more homogenous samples (e.g., age, gender, sport) across different

9 countries. Sixth and finally, our claims of universality may have been confounded by constructs

10 derived from a theory of Western origins (SDT; Deci \& Ryan, 1985). Complementary studies in

11 which indigenously derived constructs are developed and employed would significantly add to our

12 understanding of culturally specific social-psychological processes and may better account for

13 variation in the functioning of athletes around the world.

In sum, the current findings suggest that aspects of the interpersonal relationship between coaches and their athletes are aligned with athletes’ perceptions of SDT-based motivational

16 processes relevant to their well-being. Thus, good quality coach-athlete relationships can play a key

17 role in creating sport environments which support athletes realizing their potential without 
1 we can develop cross-cultural competencies relevant to all coaches who are interested in creating

2 athletic partnerships that have a positive impact on the athletes' psychological growth. 


\section{References}

Adie, J. W. \& Bartholomew, K. J. (2013). The well- and ill-being of participants in competitive sport settings: A review of motivational determinants. In C. Mohiyedinni (Ed.), Advances in the Psychology of Sport and Exercise (pp. 109-140). NY: Main Nova Publishers.

Adie, J. W., Duda, J. L., \& Ntoumanis, N. (2012). Coach autonomy support, need satisfaction and the well- and ill-being of elite youth soccer players: A longitudinal investigation. Psychology of Sport and Exercise, 13, 51-59.

Álverez, M. S., Balaguer, I., Castillo, I. \& Duda, J. L. (2009). Coach autonomy support and quality of sport engagement among young soccer players. The Spanish Journal of Psychology, 12, 138-148.

Balaguer, I., González, L., Fabra, P., Castillo, I., Mercé, J. \& Duda, J. L. (2012). Coaches' interpersonal style, basic psychological needs and the well- and ill-being of young soccer players: A longitudinal analysis. Journal of Sports Sciences, 30, 1619-1629.

Balduck, A. L., Jowett, S., \& Buelens, M. (2011). Factorial and predictive validity of the Belgian version of the CART-Q. International Journal of Sport Psychology, 42, 40-49.

Baron, R. M., \& Kenny, D. A. (1986). The moderator-mediator variable distinction in social psychological-research - Conceptual, strategic, and statistical considerations. Journal of Personality and Social Psychology, 51, 1173-1182.

Bartholomew, K. J., Ntoumanis, N., Ryan, R. M., Bosch, J., \& Thøgersen-Ntoumani, C. (2011b). Self-determination theory and diminished functioning: The role of interpersonal control and psychological need thwarting. Personality and Social Psychology Bulletin, 37, 1459-1473.

Bartholomew, K. J., Ntoumanis, N., Ryan, R. M., \& Thøgersen-Ntoumani, C. (2011a). Psychological need thwarting in the sport context: Assessing the darker side of athletic experience. Journal of Sport and Exercise Psychology, 33, 75-102.

Baumeister, R., \& Leary, M. R. (1995). The need to belong: Desire for interpersonal attachments as a fundamental human motivation. Psychological Bulletin, 117, 497-529. 
1 Bentler, P. M. (2004). EQS structural equations modeling software [Computer software] (Version 6.1). Encino, CA: Multivariate Software.

Bentler, P. M. (1990). Comparative fit index in structural models. Psychological Bulletin, 107, 238-246.

Byrne, B. M. (2006). Structural equation modeling with EQS: Basic concepts, applications, and programming (2 ed.). Mahwah, NJ: Erlbaum.

Calvo, T. G., Gimeno, E. C., Jiménez, R., Gallego, D. I., \& Murcia, J. A. M. (2010). Using self-determination theory to explain sport persistence and dropout in adolescent athletes. Spanish Journal of Psychology, 13, 677-684.

Chan, D. K., Hagger, M. S., \& Spray, C. M. (2011). Treatment motivation for rehabilitation after a sport injury: Application of the trans-contextual model. Psychology of Sport and Exercise, 12, 83-92.

Chemolli, E. \& Gagné, M. (2014), Evidence against the continuum structure underlying motivation measures derived from the self-determination theory. Psychological Assessment, 26, 575-585. doi.org/10.1037/a0036212

Choi, H., Chou, S \& Huh, J. (2013). The association between the perceived coach-athlete relationship and athletes’ basic psychological needs. Social Behavior \& Personality, 41, 1547-155.

Chirkov, V. I., Ryan, R. M., \& Willness, C. (2005). Cultural context and psychological needs in Canada and Brazil: Testing as self-determination approach to the internalization of cultural practices, identity and well-being. Journal of Cross-Cultural Psychology, 36, 423-443.

Costa, S., Ntoumanis, N., \& Bartholomew, K.J. (2015). Predicting the brighter and darker sides of interpersonal relationships: Does psychological need thwarting matter? Motivation and Emotion, 39, 11-24.

Cresswell, S L., \& Eklund, R. C. (2005). Motivation and burnout in professional rugby players. Research Quarterly for Exercise and Sport, 76, 370-376. 
deCharms, R. (1968). Personal Causation: The Internal Affective Determinants of Behavior. New York: Academic Press.

Deci, E. L., \& Ryan, R. M. (1985). Intrinsic Motivation and Self-Determination in Human Behavior. New York: Plenum Press.

Deci, E. L., \& Ryan, R. M. (1987). The support of autonomy and the control of behavior. Journal of Personality and Social Psychology, 53, 1024-1037.

Deci, E. L., \& Ryan, R. M. (2000). The “what” and “why” of goal pursuits: Human needs and the self-determination of behavior. Psychological Inquiry, 11, 227-268.

Deci, E. L., \& Ryan, R. M. (2008). Hedonia, eudaimonia, and well-being: An introduction. Journal of Happiness Studies, 9, 1-11.

Deci, E. L., Ryan, R. M. Gagné, M. Leone, D. R., Usonov, J., \& Kornazheva, B. P. (2001). Need satisfaction, motivation and well-being in organizations of a former Eastern Block country: A cross-cultural study of self-determination. Personality and Social Psychology Bulletin, 27, 930-942.

Doyal, L., \& Gough, I. (1991). A theory of human needs. New York: Guilford.

Felton, L. \& Jowett, S. (2013a). The mediating role of social environmental factors in the associations between attachment styles and basic needs satisfaction. Journal of Sport Sciences, 31, 610-628.

Felton, L., \& Jowett, S. (2013b). "What do coaches do" and "how do they relate”: Their effects on athletes’ psychological needs and functioning. Scandinavian Journal of Medicine and Sports Sciences, 23, 130-139.

Felton, L., \& Jowett, S. (2015). On understanding the role of need thwarting in the association between athlete attachment and well/ill-being. Scandinavian Journal of Medicine and Science in Sports, 25, 289-298.

Hambleton, R. K. (2005). Issues, designs, and technical guidelines for adapting tests into multiple languages and cultures. In R. K. Hambleton, P. Merenda \& C. Spielberger (Eds.), Adapting 
educational and psychological tests for cross-cultural assessment (pp. 3-38). Mahwah, NJ: Lawrence Erlbaum.

Hofstede, G. (2001). Culture’s consequences: Comparing values, behaviors, institutions, and organizations across nations ( $2^{\text {nd }}$ Ed.). Thousand Oaks, CA: Sage.

Hu, L., \& Bentler, P. M. (1999). Cutoff criteria for fit indexes in covariance structural analysis: Conventional criteria versus new alternatives. Structural Equation Modeling, 6, 1-55.

Jowett, S. (2005). On repairing and enhancing the coach-athlete relationship. In S. Jowett \& M. Jones (Eds.), The psychology of coaching. Leicester: The British Psychological Society.

Jowett, S. (2007). Interdependence analysis and the 3+1Cs in the coach-athlete relationship. In S. Jowett, \& D. Lavalle (Eds.), Social psychology in sport (pp.15e28). IL: Human Kinetics.

Jowett, S. (in press). Coaching Effectiveness: The Coach-Athlete Relationship at its Heart. Current Opinion in Psychology.

Jowett, S., \& Nezlek, J. (2012). Relationship Interdependence and Satisfaction with Important Outcomes in Coach-Athlete Dyads. Journal of Social and Personal Relationships, 29, 287-301.

Jowett, S., \& Ntoumanis, N. (2004). The Coach-Athlete Relationship Questionnaire (CART-Q): Development and initial validation. Scandinavian Journal of Medicine and Science in Sports, 14, 245-257.

Jowett, S., \& Shanmugam, V. (2016). Relational Coaching in Sport: Its psychological underpinnings and practical effectiveness. In R. Schinke, K.R. McGannon, \& B. Smith (Eds), Routledge International Handbook of Sport Psychology. London: Routledge.

Kagitcibasi, C. (1996). The autonomous-relational self: A new synthesis. European Psychologist, 1, 180-186.

Kelley, H. H., \& Thibaut, J. W. (1978). Interpersonal Relations: A Theory of Interdependence. New York: Wiley.

Kowal, J., \& Fortier, M. S. (1999) Motivational determinants of flow: Contributions from self-determination theory. Journal of Social Psychology, 139, 355-368. 
1 La Guardia, J. G. \& Patrick, H. (2008). Self-determination theory as a fundamental theory of close relationships. Canadian Psychology, 49, 201-209.

La Guardia, J. G., Ryan, R. M., Couchman, C. E., \& Deci, E. L. (2000). Within-person variation in security of attachment: A self-determination theory perspective on attachment, need fulfillment, and well-being. Journal of Personality and Social Psychology, 79, 367-384.

Lonsdale, C., Hodge, K., \& Rose, E. (2009). Athlete burnout in elite sport: A self-determination perspective. Journal of Sports Sciences, 27, 785-795.

Lonsdale, C., Hodge, K., \& Rose, E. A. (2008). The Behavioral Regulation in Sport Questionnaire (BRSQ): Instrument development and initial validity evidence. Journal of Sport \& Exercise Psychology, 30, 323-355.

Lorimer, R. \& Jowett, S. (2013). Empathic understanding and accuracy in the coach-athlete relationship. In J. Denison, W. Gilbert, \& P. Potrac (Eds.). Routledge Handbook of Sports Coaching (321-332). London: Routledge.

Mageau, G. A., \& Vallerand, R. J. (2003). The coach-athlete relationship: A motivational model. Journal of Sport Sciences, 21, 883-904.

Mallett, C. J. (2005). Self-determination theory: A case study of evidence-based coaching. The Sport Psychologist, 19, 417-429.

Marsh, H. W., Marco, I. T., \& Abcy, F. H. (2002). Cross-cultural validity of the physical self-description questionnaire: comparison of factor structures in Australia, Spain, and Turkey. Research Quarterly for Exercise and Sport, 73, 257-270.

Miller, J. G. (1999). Cultural conceptions of duty: Implications for motivation and morality. In D. Munroe, J. F. Schumaker, \& S. C. Carr (Eds.), Motivation and culture (pp. 178-192). New York: Routledge.

Oishi, S. (2000). Goals as cornerstones of subjective well-being: Linking individuals and cultures. In E. Diener \& E. M. Suh (Eds.), Culture and subjective well-being (pp. 87-112). Cambridge, MA: Bradford. 
1 Pelletier, L. G., Fortier, M. S., Vallerand, R. J., \& Brière, N. M. (2001). Associations among perceived autonomy support, forms of self-regulation, and persistence: A prospective study. Motivation and Emotion, 25, 279-306.

Pope, P J. \& Wilson, P. M. (2012). Understanding motivational processes in university rugby players: A preliminary test of the hierarchical model of intrinsic and extrinsic motivation at the contextual level. International Journal of Sport Science and Coaching, 7, 89-107.

Quested, E. \& Duda, J. L. (2010). Exploring the social-environmental determinants of well- and ill-being in dancers: A test of basic needs theory. Journal of Sport and Exercise Psychology, 32, 39-60.

Reinboth, M., Duda, J. L., \& Ntoumanis, N. (2004). Dimensions of coaching behavior, need satisfaction, and the psychological and physical welfare of young athletes. Motivation and Emotion, 28, 297-313.

Rhind, D., Jowett, S., \& Yang, S. (2012). A comparison of athletes' perceptions of the coach-athlete relationship in team and individual sports. Journal of Sport Behavior, 35, 433-441.

Riley, A., \& Smith, A.L. (2011). Perceived coach-athlete and peer relationships of young athletes and self-determined motivation for sport. International Journal of Sport Psychology, 42, 115-133.

Ryan, R. M., \& Deci, E. L. (2000). Self-determination theory and the facilitation of intrinsic motivation, social development, and well-being. American Psychologist, 55, 68-78.

Ryan, R.M., \& Deci, E. L. (2002). An overview of self-determination theory: An organismic dialectical perspective. In Deci, E. L., \& Ryan, R. (Eds.), Handbook of Self-Determination (pp. 3-37). Rochester, NY: University of Rochester Press.

Ryan, R. M., \& Deci, E. L. (2007). Active human nature: Self-determination theory and the promotion and maintenance of sport, exercise, and health. In M. S. Hagger \& N. L. D. Chatzisarantis (Eds.), Intrinsic motivation and self-determination in exercise and sport (pp. 1-19). Champaign, IL: Human Kinetics. 
Sarrazin, P., Vallerand, R. J., Guillet, E., Pelletier, L. G. and Cury, F. (2003). Motivation and Dropout in Female Handballers: A 21 Month Prospective Study. European Journal of Social Psychology, 32, 395-418.

Satorra, A., \& Bentler, P. M. (1988). Scaling corrections for chi-square statistics in covariance structure analysis. ASA Proceedings of the Business and Economic Section, 308-313.

Sheldon, K. M., Elliot, A. J., Ryan, R. M., Chirkov,V. I., Kim,Y.,Wu, C., et al. (2004). Self-concordance and subjective well-being in four cultures. Journal of Cross-Cultural Psychology, 35, 209-223.

Sheldon, K. M., Osin, E., Gordeeva, T. O., Suchkov, D. D., Bobrov, V. V., Rasskazova, E. I., Sychev, O. A. (November 30, 2015). Evaluating the Dimensionality of the Relative Autonomy Continuum in US and Russian Samples. Higher School of Economics Research Paper No. WP BRP 48/PSY/2015. Available at SSRN: https://ssrn.com/abstract=2696937 or http://dx.doi.org/10.2139/ssrn.2696937

Taylor, I. M (2015). The five self-determination mini-theories applied to sport. In S. D. Mellalieu \& S. Hanton (Eds.). Contemporary Advances in Sport Psychology (pp. 68-90). Abingdon, Oxon: Routledge

Taylor, I. M. \& Lonsdale, C. (2010). Cultural differences in the relationships between autonomy support, psychological need satisfaction, subjective vitality, and effort in British and Chinese physical education. Journal of Sport and Exercise Psychology, 32, 655-673.

Tennant, R., Hiller, L., Fishwick, R., Platt, S., Joseph, S., Weich, S., et al. (2007). The Warwick-Edinburgh mental well-being scale (WEMWBS): development and UK validation. Health and Quality of Life Outcomes, 5, 1-13.

Vallerand, R. J. (2007). A hierarchical model of intrinsic and extrinsic motivation for sport and physical activity. In M. S. Hagger, \& N. L. D. Chatzisarantis (Eds.), Self-Determination Theory in Exercise and Sport (pp. 255-279). Champaign, IL: Human Kinetics.

White, R. W. (1959). Motivation reconsidered: The concept of competence. Psychological Review, 

66, 297-333.

2 Yang, S., \& Jowett, S. (2012). Psychometric Properties of the Coach-Athlete Relationship Questionnaire (CART-Q) in Seven Counties. Psychology of Sport and Exercise, 13(1), 36-43.

4 Zhao, X. S., Lynch, J. G., \& Chen, Q. M. (2010). Reconsidering Baron and Kenny: Myths and truths about mediation analysis. Journal of Consumer Research, 37, 197-206. 